REFLUXKRANKHEIT

\section{Berufsleiden der Opernsänger}

- Opernsänger sind offenbar eine Risikogruppe für die Refluxkrankheit, erklärte Prof. Stephan Miehlke, Dresden. Die Erklärung ist einfach: Die täglichen und jahrelangen Gesangsübungen führen durch den regelmäßigen Einsatz des Zwerchfells zu einem abrupten und prolongierten Anstieg des intraabdominellen

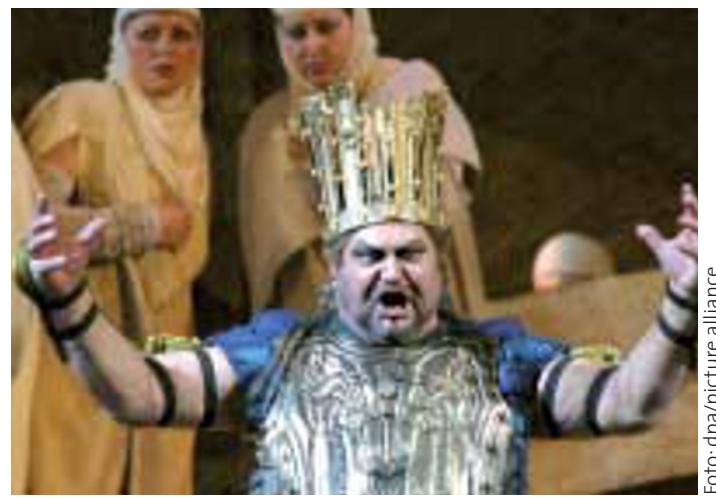

Belcanto mit saurem Nachgeschmack. Drucks, was auf Dauer zu einer Schwächung des diaphragmalen Sphinkters, der eine Antirefluxbarriere darstellt, führen kann. „Man muss davon ausgehen, dass manche Opernsänger ihren Beruf aufgrund dieser belästigenden Symptome aufgeben müssen," sagte Miehlke. Bevor die Refluxkrankheit jedoch offiziell als Berufskrankheit für hauptberufliche Sänger anerkannt werden könne, seien weitere Studien mit pH-Messungen und Manometrie im Ösophagus erforderlich.

\title{
GASTROINTESTINALE KARZINOME
}

\section{Bessere Überlebensraten durch Psychotherapie}

— In einer randomisierten kontrollierten Studie wurde bei 271 Patienten mit gastrointestinalen Karzinomen der Nutzen einer begleitenden Psychotherapie während des Krankenhausaufenthalts evaluiert. Im Vergleich zur Kontrollgruppe fand sich, so Prof. Wolfgang Fischbach, Aschaffenburg, ein signifikant besseres Überleben nach zehn Jahren bei den psychotherapeutisch behandelten Patienten. Die
Psychotherapie erwies sich als ein unabhängiger prognostischer Faktor, d. h. der Nutzen war unabhängig vom TNM-Stadium, dem Vorliegen eines Residualtumors und der Tumorlokalisation. Dieser therapeutische Gewinn konnte mit relativ geringem Zeitaufwand erreicht werden: Die betroffenen Patienten wurden nur ca. sieben Stunden während des Klinikaufenthalts psychotherapeutisch behandelt.

\section{PROPHYLAXE DES MAGENKARZINOMS}

\section{Keine H.-pylori-Eradikation für alle}

- Epidemiologische und experimentelle Daten, Observations- und Interventionsstudien sprechen eindeutig dafür, dass eine kausale Assoziation zwischen Helicobacter pylori und dem Magenkarzinom besteht. Doch trotz der überzeugenden Evidenz wird, so W. Fischbach, ein allgemeines Screening nach $\mathrm{H}$. pylori und eine prophylaktische Eradikation bisher nicht empfohlen. Als Gründe für diese Zurückhaltung

\footnotetext{
- Quelle: Gastro Update 2008, Wiesbaden, 22.-23.2.2008
}

nannte er das niedrige Lebenszeitrisiko für ein Magenkarzinom bei mit $\mathrm{H}$. pylori infizierten Personen und die Sorge vor einer Resistenzentwicklung bei breiter Antibiotikaanwendung. Auch fehle bisher der eindeutige Nachweis, dass durch präventive Keimeradikation die Karzinominzidenz tatsächlich gesenkt werden könne. Deshalb wird sich, so Fischbach, die H.-pylori-Eradikation zur Magenkarzinomprophylaxe auch künftig auf Risikopersonen wie z. B. Patienten mit Magenkarzinom in der Familienanamnese beschränken.
PANKREATITIS DURCH MEDIKAMENTE

\section{Entwarnung für Diuretika}

- Antihypertensiva, insbesondere Diuretika, gehören zu den Medikamenten, die oft als Ursache einer akuten Pankreatitis angeschuldigt werden. Nach einer neueren Studie ist die Gabe eines Diuretikums jedoch nicht mit einem erhöhten Pankreatitisrisiko assoziiert, betonte Prof. Peter Layer, Hamburg. Dies gilt nach seinen Worten für alle Diuretikagruppen. Ein leicht erhöhtes Risiko konnte für Kalziumantagonisten und ACE-Hemmer, allerdings nur in den ersten Monaten nach Therapiebeginn dokumentiert werden.

Auch Hormonpräparate sind im Hinblick auf ihr Pankreatitisrisiko hin untersucht worden. Bei Patientinnen, die eine postmenopausale Hormonersatztherapie erhielten, wurde diesbezüglich kein erhöhtes Risiko nachgewiesen.

\section{LANGZEITTHERAPIE MIT PPI}

\section{Magenschutz versus Frakturrisiko}

- Dass eine Langzeittherapie mit Protonenpumpenhemmern (PPI) das Risiko für eine Hüftfraktur signifikant erhöht, ist nach dem Ergebnis einer neueren Studie eindeutig mit „Ja“ zu beantworten. Allerdings muss immer das individuelle Nutzen-Risiko-Verhältnis abgeschätzt werden. Wie Prof. S. Miehlke ausführte, dürfte dieses bei Patienten mit Refluxkrankheit und auch bei älteren Patienten im Hinblick auf die Prävention von NSAR-Ulkus-Komplikationen in der Regel positiv ausfallen. Um das Risiko so niedrig wie möglich zu halten, ist die korrekte Indikationsstellung und das Anstreben einer möglichst niedrigen Dosierung wichtig, z. B. durch eine Step-down-Therapie bei Reflux.

Als Erklärung für das erhöhte Hüftfrakturrisiko wird eine durch die medikamentös induzierte Hypochlorhydrie verminderte intestinale Kalziumresorption angenommen. Deshalb wird diskutiert, ob ältere Patienten mit hoch dosierter Langzeit-PPITherapie eine Kalziumsubstitution erhalten sollten.

STI = 